\title{
QUALITATIVE STUDY ON THE APPLICATIONS OF SMARTPHONES IN MEDICAL RESEARCH
}

\author{
L Abeynaike BSc ${ }^{1}$ \\ ${ }^{1}$ Centre for Inflammatory Diseases, Department of Medicine, Monash University, Melbourne, Australia. \\ Corresponding author: latasha.abeynaike@monash.edu
}

Background: Mobile technology advancements have bought many benefits to professionals across all industries, particularly an increase in productivity. There is a paucity of information regarding the use of smartphone applications in medical research, particularly within the laboratory has not been examined.

Method: We performed a qualitative study on the use of smartphones in the laboratory. Medical researchers were surveyed regarding use of their smartphones in the laboratory, as well as the use of apps, or "applications". We also performed analysis on available applications and current standing of smartphone integration with laboratory equipment.

Results: Survey responses indicated researchers to be more productive as a result of owning smartphones, however most did not utilise applications, and none utilised field-specific applications in the laboratory. Several issues preventing researchers from using their smartphones and applications in the laboratory were identified. These included occupational health and safety reasons, security, potential for distraction, absence of wireless connectivity, and a lack of awareness. Analysis of iPhone applications demonstrated availability of a range of reference and functional applications performing essential laboratory tasks.

Conclusion: This study demonstrates that smartphones are currently not used to their full potential within the laboratory, and there are hurdles to overcome before wider use in the field of medical research. However as time and technology progresses further, and with increasing integration of smartphone technology with current laboratory equipment, researchers will embrace the smartphone as a costeffective and timesaving device.

Journal MTM 1:1:19-23, 2012 doi:10.7309/jmtm.7 www.journalmtm.com

\section{Introduction}

Smartphones have proven to be powerful tools in the workplace, increasing connectivity and productivity. Some of the benefits in the workplace are seen as negative as the smartphone can be complex and distracting. The prospect of being able to be contacted at anytime is often seen as an invasion of personal time. Despite this, smartphones have a function in all aspects of life, and their use is increasing dramatically.
The smartphone is considered different to other mobile phones due to the presence of advanced computing systems, notably with high-speed internet access via wi-fi and mobile broadband. With constantly evolving features, such as large touch screens, QWERTY keyboards, web browsers, inbuilt cameras, Global positioning systems (GPS) and voice command, smartphones have become powerful and essential tools. In addition, smartphones have available to them a vast range of applications which can be downloaded onto the smartphone from the 
Fra JMTM

internet. A global study in 2011 conducted by Google ${ }^{1}{ }^{1}$ demonstrates $37 \%$ of Australians utilise smartphones; they have an average of 25 applications on their smartphone, including 8 paid applications. Much of the growth in smartphone use is attributed to the Apple ${ }^{\circledR}$ iPhone, which is used by $46 \%$ of surveyed smartphone users globally.

Organisations and individuals have developed applications, which allow people to be more efficient and flexible; to perform tasks on-the-go where they would normally require a computer. The smartphone's ability to handle complex programming has allowed the generation of applications which perform highly specific functions for a range of professionals. Within the medical field, an ample quantity of applications exist, including diagnostic protocols, guidelines, and drug reference applications for clinicians across all disciplines of medicine. Applications also exist which allow patient monitoring, where patients are able to $\log$ in data or symptoms online. For example, the Apple iPhone accelerometer allows measurement and monitoring of tremors for patients with Parkinson's disease from any location ${ }^{2}$, arguably more accurately than previous methods within the doctors office.

There are many applications available for medical researchers in the laboratory. They provide access to a wide range of information ranging from reference guides for molecules, chemicals, medical journals and databases, to laboratory technique guides and calculators. However, the use of the smartphone specifically by medical researchers in the laboratory has never been examined.

Therefore, the aim of this study was to investigate the utilisation of smartphones in the field of medical research by performing a qualitative survey. To the best of our knowledge this study is the first to examine issues encountered with smartphone use in the laboratory, and applications usage. In addition, we discuss the availability of applications for the Apple iPhone for use in the laboratory.

\section{Methods}

An online survey was generated consisting of 10 questions, with a combination of multi choice and discussion responses required. Being a qualitative study, a total of five surveys were completed anonymously. In conjunction with the survey, three researchers were interviewed. Researchers consisted of research assistants, post doctoral and postgraduate ( $\mathrm{PhD}$ in Medicine) staff covering a range of skills and experience in non-clinical medical research at Monash University, Clayton, Australia. The survey questions were structured to include individuals that did not use smartphones, as well as those that may

\section{ORIGINAL ARTICLE}

use applications. Responses were analysed and key issues were extracted and discussed. Interviewed researchers were asked to elaborate on their issues with smartphones in the laboratory and what they would consider a useful application.

Information on available applications for the Apple iPhone, the predominant smartphone used by surveyed staff as well as in Australia, was gathered by searching scientific forums and websites, and by entering a range of key words in the Apple iTunes Application store, including laboratory techniques and field-specific words. Several applications were downloaded and used on an iPhone 4S, including multiple applications performing similar tasks, and their functions and usability were compared.

\section{Results}

All surveyed and interviewed researchers owned smartphones; with the leading smartphone being the Apple iPhone. Researchers classified themselves as more productive as a result of owning a smartphone. Specifically, researchers indicate that accessing email, calendars and to-do lists, contacting colleagues and transferring data were the ways in which the smartphone was most beneficial to them at work. Only 2 of the 5 surveyed researchers utilised applications; none of which were field-specific. These applications simply consisted of a timer and basic calculator. Further, these functions were utilised during experimental planning, but only occasionally within the laboratory.

\section{Limitations for smartphone use in the laboratory}

Scientists surveyed sometimes, rarely or never carry their smartphone into the laboratory. Survey responses regarding reasons why researchers do not carry their smartphone into the laboratory or use applications were analysed and themes were evident.

Occupational health and safety

Concern for occupational health and safety was a major issue for most researchers. Laboratory work involving bio-hazardous substances such as human or animal fluids and tissues, and corrosive chemicals do not allow a safe environment for any personal belongings. Survey responses indicated that the removal of gloves and protective gear to access a phone can be troublesome and disruptive during experiments.

\section{Security}

Some researchers claimed that their laboratory space is not secure enough to leave personal belongings; 
Fra JMTM

and often not having pockets on clothing means the phone cannot be carried personally.

\section{Distractions}

Researchers also state that having their smartphone in the laboratory with them can be distracting. This includes distractions with phone calls, text messages and email notifications as well as social networking sites and games.

Absence of wireless network within the laboratory The particular building in which surveyed researcher's laboratories were located did not provide a wireless network. An intranet system containing staff contact details was suggested by one researcher to be a potentially convenient function to increase connectivity within the workplace.

\section{Lack of awareness}

One issue faced when searching for applications for this study, was the difficulty in finding themes the correct key words had to be used. Some researchers suggested the need for applications within the survey, which unaware to them, already existed. For example, one researcher stated:

"Perhaps a molecular weight of commonly used materials, and MSDS(Material Safety Data Sheets) /hazards list"

Following this comment, we entered keywords from the above statement and found applications for both suggestions.

\section{Other issues}

Other reasons for which the smartphone and applications were not utilised within the laboratory include the cost of the applications, however this was not a major comment. Some researchers indicated that no applications exist for their specific needs.

As only a few surveyed staff were interested in applications, minimal suggestions were made as to the functions they would like to see in them. It was suggested by one researcher that an application containing an experimental protocol template which could be filled out quickly within the laboratory would be useful, and exported later via email. No applications currently exist which aid in the planning and writing up of experimental protocols. Another researcher indicated functions that are not already performed by other equipment, such as timers, cell counters and calculators would be useful:

\section{ORIGINAL ARTICLE}

\begin{abstract}
"If there was something particularly unique that I couldn't use other equipment for when I'm in the laboratory."
\end{abstract}

A few researchers stated that they were happy with their current methods and did not think that an application would be beneficial to them.

\section{Discussion}

While researchers state that their smartphones have made them more productive in the work place by making them more organised, this is independent of applications. From this study, it appears that the benefits of smartphones and applications do not necessarily outweigh the negatives for researchers.

The limitations discussed may have solutions to overcome them. Upon examining the issues stated by researchers, it became apparent that research institutes and workplaces need to adapt to the increasing use of smartphones by their researchers. Surveyed researchers were unaware of available applications, indicating workplace involvement to increase awareness of field-relevant applications is required. Promoting increased security within the laboratory, such as restricting access to laboratories via card- only access or personal lockable drawers or lockers and increasing awareness for occupational health and safety rules regarding safe areas for personal belongings could allow researchers to carry their smartphones into the laboratory more often. Waterproof protective casing for smartphones or lanyard attachment would allow researchers to use their phones and comply with experimental conditions. A recent editorial mentioned the possibility of a removable skin.3Furthermore, providing information on how to manage settings on smartphones to restrict notifications for emails and phone calls and social networking sites may reduce distractions in the laboratory.

Wireless internet connections allow access to parts of buildings such as basements in which service provider broadband networks are not accessible. Particularly where smartphone plans or contracts are not covered by employers, researchers may be reluctant to access the internet for work purposes. Further, an intranet network for staff to access useful links such as journals, reference guides, calculators relevant to their field of research and staff contacts may be beneficial for all smartphone researchers.

Many people who own smartphones are not necessarily technically knowledgeable; and with smartphones now being more affordable, many people own smartphones with little interest in most of 


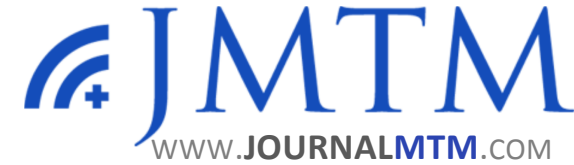

its functions. Some researchers indicated that they were satisfied with their current techniques. For some, the complexity of the smartphone is daunting. However, as time and technology advance, many will 'fall behind' and miss out on the many advantages of owning a smartphone, and therefore may benefit from some encouragement from employers and colleagues.

A range of applications are available for researchers covering everyday laboratory tasks. Here we discuss some applications found available for the iPhone. Life Technologies have developed a free application providing all the essential calculators- molarity, dilutions and unit conversions. Celeromics' ${ }^{\circledR}$ Cell Count application allows continuous counting of squares, viability percentages and nice large easy-totap buttons on screen. Several laboratory timer applications are available, ranging from no cost to a few dollars. Major biotechnology company Sigma Aldrich ${ }^{\circledR}$ have produced a slightly cumbersome 6timer application, in contrast much simpler timers exist providing quick access buttons ideal for hectic experiments. Applications covering more specific laboratory techniques are covered, including Real time- Polymerase Chain Reaction (PCR). Bio-Rad ${ }^{\circledR}$ real-Time PCR is an easy to use interactive guide to the technique. BioLegend $\mathbb{R}$ have developed a range of reference applications, including a cytokine and chemokine guide for both mouse and human. They have also developed a flow cytometry guide application, which includes a fluorochrome guide, as well as a timer and antibody calculator.

Reference guides are available in the form of applications, useful both inside and outside the laboratory. Particularly useful is the MSDS (Material Safety Data Sheets) available in the Chemical Safety Data Sheets application; this application in conjunction with a text recognition system would be highly convenient. ${ }^{3}$ Genetic code contains nucleic acid and amino acid properties. Many scientific and medical journals have released applications intended for medical professionals, including The New England Journal of Medicine, Nature Publishing Group and databases such as Science-Direct, particularly useful with an institution subscription allowing access to any journal or book.

A new aspect of research dedicated to combining the functions of laboratory equipment with the convenience of the mobile phone is now well underway. The replacement of with the camera phone is now a possibility, with a study utilising the phone camera function to detect Enzyme linked immunesorbent assay (ELISA) results. ${ }^{4}$ A large range of studies exist examining the combination of microscopy components with the mobile phone, including fluorescence microscopy. ${ }^{5-8}$ Hardware attachments to the phone allowing flow cytometry functions have also been explore. ${ }^{9}$ Using the smartphone to store and transfer data is also a useful function in the laboratory, including those located in remote areas, where microscopic images can be sent for analysis or diagnosis immediately. ${ }^{10}$

Upon analysis of the above applications available for the medical researcher, it is clear that applications can provide many benefits for a researcher particularly regarding time management and productivity both inside and outside the laboratory. The primary advantage is that multiple functions are conveniently available in one device. Most of the analysed applications were free of charge, and the most expensive applications were less than five dollars (Australian currency). For the more sophisticated functions, significant benefits exist to the laboratory. Complex machines such as spectrometers, flow cytometers and microscopes with their associated computer systems are a large financial burden on laboratories. The prospect of smartphone applications or hardware attachments would save money and space, and be particularly beneficial to lower income laboratories. $^{6}$

\section{Conclusion}

This study demonstrates that while researchers believe that smartphones have the capability to increase productivity at work. However, some limitations apply in the laboratory. Upon analysis of survey responses it appears that researchers have the potential to be even more productive with a smartphone. However, there needs to be increased involvement with employers and a heightened awareness of its potential. With the emphasis on the 'hands free' utilisation of smartphones, and the plethora of novel research examining the integration of complex laboratory equipment components, the future of smartphone usage in the laboratory is indeed promising.

\section{References}

1. IPOS Research, Google. Australia Mobile Smartphone Consumer Study, 2011.

2. Implementation of an iPhone for characterizing Parkinson's disease tremor through a wireless accelerometer application. Engineering in Medicine and Biology Society 
F. JMTM

(EMBC), 2010 Annual International Conference of the IEEE; 2010 Aug. 31 2010-Sept. 42010.

3. Anon. The scientist and the smartphone. Nat Meth 2010;7(2):87-87.

4. Wang S, Zhao X, Khimji I, Akbas R, Qiu W, Edwards D, et al. Integration of cell phone imaging with microchip ELISA to detect ovarian cancer HE4 biomarker in urine at the point-of-care. Lab on a Chip 2011;11(20).

5. Breslauer DN, Maamari RN, Switz NA, Lam WA, Fletcher DA. Mobile Phone Based Clinical Microscopy for Global Health Applications. PLoS ONE 2009;4(7):e6320.

6. Tuijn CJ, Hoefman BJ, van Beijma H, Oskam L, evrollier N. Data and Image Transfer Using Mobile phones to Strengthen Microscopy-Based Diagnostic Services in Low and Middle Income Country Laboratories. PLoS ONE 2011;6(12).

7. Zhu H, Yaglidere O, Su TW, Tseng D, Ozcan A. Costeffective and compact wide-field fluorescent imaging on a cell-phone. Lab Chip 2011;11(2):315-22.

\section{ORIGINAL ARTICLE}

8. Tseng D, Mudanyali O, Oztoprak C, Isikman SO, Sencan I, Yaglidere O, et al. Lensfree microscopy on a cellphone. Lab Chip 2010;10(14):1787-92.

9. Zhu H, Mavandadi S, Coskun AF, Yaglidere O, Ozcan A. Optofluidic fluorescent imaging cytometry on a cell phone. Anal Chem 2011;83(17):6641-7.

10. Bellina L, Missoni E. Mobile cell-phones (M-phones) in telemicroscopy: increasing connectivity of isolated laboratories. Diagn Pathol 2009;4:19.

\section{Disclaimer}

All product names, logos, brands and other trademarks featured or referred to within the Journal of Mobile Technology in Medicine e-publication remain the property of their respective trademark holders.

These trademark holders are not affiliated with the Journal of Mobile Technology in Medicine, and do not sponsor or endorse this journal. 